\title{
Heterocyclic synthesis with nitriles: Synthesis of pyrazolopyrimidine and pyrazolopyridine derivatives
}

\author{
Abdellatif M. Salaheldin*, Ana M. F. Oliveira-Campos and Lígia M. Rodrigues \\ Centro de Química, IBQF, Universidade do Minho, Campus de Gualtar, 4710-057 Braga, Portugal \\ salaheldin@quimica.uminho.pt
}

\begin{abstract}
The reaction of $N_{1}$-substituted-5-amino-4-cyanopyrazoles with malononitrile and diethylmalonate occurs with formation of 6-substituted pyrazolo[3,4-d]pyrimidines, and pyrazolo[3,4-b]pyridines respectively. The structures of the products and conceivable mechanisms are discussed.
\end{abstract}

Keywords: Aminopyrazole, pyrazolo[3,4- $d]$ pyrimidines, pyrazolo[3,4-b]pyridines, diethylmalonate, malononitrile.

\section{INTRODUCTION}

Pyrazolopyridines, pyrazolopyrimidines and related fused heterocycles are of interest as potential bioactive molecules. Pyrazolo[3,4- $d$ ] pyrimidines were identified as a general class of adenosine receptors, ${ }^{[1-3]}$ due to the similarity between their structures and purines. Pyrazolo[3,4-b]pyridines are also important compounds as a result of their biological activity and structural relationship to azaindoles. A number of pyrazolo[3,4- $b]$ pyridines are potentially biologically active compounds as new inhibitors of xantine oxidase. ${ }^{[4,5]}$ Because of this wide range of activities, we have been interested on orthoaminocyanopyrazoles ${ }^{[5,6]}$ or their derivatives as inhibitors of xanthine oxidase. ${ }^{[7]}$ For this purpose we started from the key intermediates 1a-d ( $N_{1}$-substituted-5-amino-4cyanopyrazoles) 1 (Scheme 1) and reacted them with malononitrile and diethylmalonate to obtain pyrazolo[3,4- $d]$ pyrimidines and pyrazolo[3,4-b]pyridines respectively.

\section{RESULTS AND DISCUSSION}

With the aim of obtaining condensed pyrazolo[3,4- $d$ ]pyrimidine and pyrazolo[3,4-b] pyridine systems the condensation has been carried out of a substituted aminopyrazole, which contains a cyano group in the ortho position, with malononitrile and diethylmalonate. 
The 5-amino-1-substitutedpyrazole-4-carbonitriles 1, were used as starting materials, they contain an amino and a cyano group in adjacent positions, which is required for the synthesis of the condensed systems including pyridine and pyrimidine.

It has been found that reaction of compound 1a with malononitrile in refluxing ethanol in the presence of triethylamine afforded a yellow crystalline solid of mp $254-256^{\circ} \mathrm{C}$. It was expected that this reaction would give the pyrazolopyrimidine $4 \mathbf{a}$ or pyrazolopyridine 5a via the intermediate 3a. However, the micro-analytical data showed that this product has the molecular formula $\mathrm{C}_{20} \mathrm{H}_{16} \mathrm{~N}_{8}$. Furthermore, the mass spectrum (EI) of this product showed a molecular ion at $\mathrm{m} / \mathrm{z}=368$ and the IR spectrum displays an absorption at 3463 $\mathrm{cm}^{-1}$, corresponding to $\mathrm{NH}_{2}$ stretching, and no $\mathrm{CN}$ absorption. The ${ }^{1} \mathrm{H}$ NMR spectrum reveals two singlets for the amino groups at 5.44 and $5.88 \mathrm{ppm}$ and two singlets for the pyrazole H-3 protons. Structure 2a was thus suggested for this product. The formation of compound 2a may be envisaged via initial condensation of the amino group of one molecule of the $o$-aminonitrile with the cyano group of a second molecule to give an intermediate amidine which then undergoes a second, but intramolecular, amine-nitrile condensation to give the isolated product. To confirm this hypothesis, reflux of compound 1a in ethanol and triethylamine afforded a product completely identical to $\mathbf{2 a}$. A similar result had been established by Taylor and Borror in the formation of $2 \mathbf{a}$ (Scheme 1). ${ }^{[8]}$

Compounds 1b-d were refluxed under the same reaction conditions to afford $\mathbf{2 b}-\mathbf{d}$.

In ethanolic sodium ethoxide solution, compounds $\mathbf{1 a}$ and $\mathbf{1 b}$ reacted with malononitrile to afford white powders of $\mathrm{mp} 242-244^{\circ} \mathrm{C}$ for $\mathbf{4 a}$ and $296-298^{\circ} \mathrm{C}$ for $\mathbf{4 b}$, respectively. The ${ }^{1} \mathrm{H}$ NMR spectrum of compound $4 \mathbf{a}$ revealed a methylene singlet at $\delta 4.17 \mathrm{ppm}$ and pyrazole $\mathrm{H}-3$ as a singlet at $8.34 \mathrm{ppm}$ besides other signals attributable to an aromatic compound and only one $\mathrm{NH}_{2}$ group at $8.0 \mathrm{ppm}$ as expected (cf. Experimental). Based on these data it seemed that a $-\mathrm{CH}_{2} \mathrm{CN}$ side chain is present and that the cyclization took place by addition of the $\mathrm{NH}_{2}$ in the pyrazole 1 to the $\mathrm{CN}$ of the malononitrile to form the amidine intermediate 3 followed by an attack of the newly formed amino group to the $\mathrm{CN}$ of $\mathbf{1}$ to afford the pyrazolopyrimidine $\mathbf{4}$ and not the pyrazolopyridine $\mathbf{5}$ as shown in Scheme 1. Similar cyclizations with other nitriles have been reported. ${ }^{[9]}$ 
On the contrary, reaction of compounds 1a,b with diethylmalonate in ethanolic sodium ethoxide solution gave pyrazolopyrimidines $\mathbf{6 a}, \mathbf{b}$. The structure of compounds $\mathbf{6}$ was confirmed by mass and NMR spectroscopic data. The ${ }^{1} \mathrm{H}$ NMR spectrum of compound 6a revealed the ester group as a triplet for the $\mathrm{CH}_{3}$ protons at $1.33 \mathrm{ppm}$ and a quartet for the $\mathrm{CH}_{2}$ protons at $4.40 \mathrm{ppm}$, besides other signals assigned to aromatics, pyrazole $\mathrm{H}-3$, one $\mathrm{NH}_{2}$ group, and an $\mathrm{OH}$ signal at $12.31 \mathrm{ppm}$ (Scheme 1). ${ }^{[10]}$

In an attempt to introduce a formyl group at position 3 in pyrazole $\mathbf{1}$, aminopyrazole $\mathbf{1}$ was reacted with Vilsmeier reagent (DMF-POCl $\mathrm{C}_{3}$ ) at $70^{\circ} \mathrm{C}$ for $3 \mathrm{~h}$. To the product which was obtained structure $\mathbf{8}$ was proposed based on the NMR data which indicate the presence of $\mathrm{OH}$ group and the pyrazole $\mathrm{H}-3$, the reaction proceeding via the intermediacy of 7 (Scheme 2). The structure of compound $\mathbf{8 b}$ could be confirmed by an alternative synthesis, as described before, converting $\mathbf{1 b}$ to the amide $\mathbf{9}$, by treatment with cold concentrated sulfuric acid, followed by boiling compound 9, mp 202-204 ${ }^{\circ} \mathrm{C}$ (Lit. mp $\left.204-205^{\circ} \mathrm{C}\right)^{[11]}$, in formamide. The product isolated was the 4-hydroxypyrazolo[3,4d]pyrimidine $\mathbf{8 b}$, whose spectral characteristics were completely coincident with the sample obtained before (Scheme 2). 


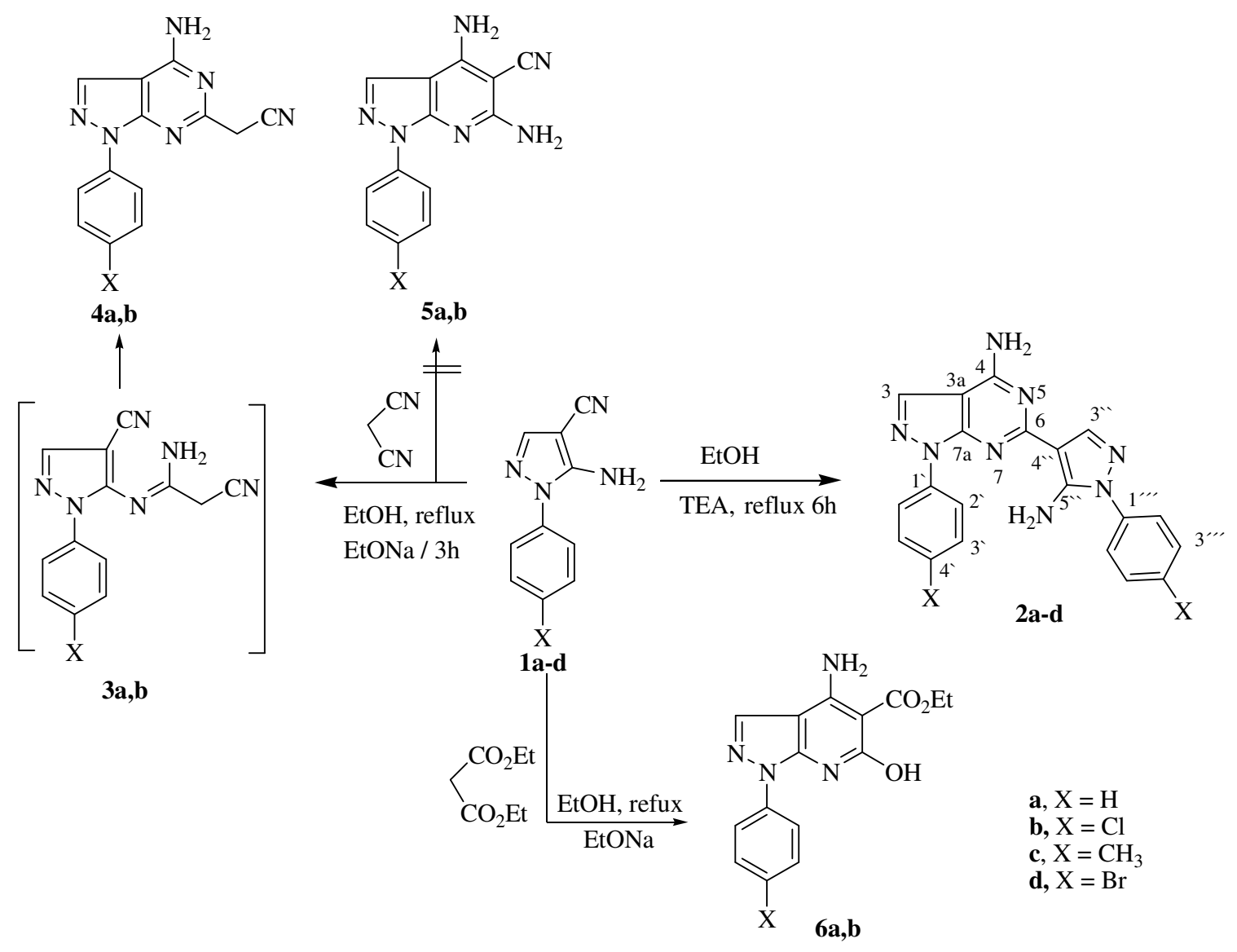

Scheme 1

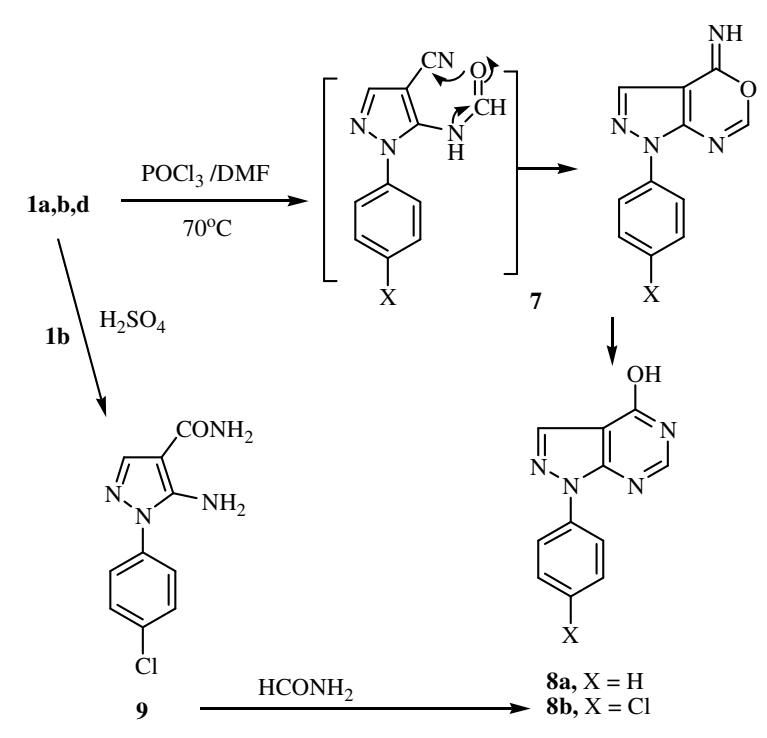

Scheme 2 
The compound $\mathbf{8 b}$, 4-hydroxypyrazolopyrimidine, was easily converted to the corresponding 4-chloropyrazolopyrimidine, the precursor to the 4-substituted aminopyrazolopyrimidine, as we described recently [6].

\section{EXPERIMENTAL}

General Procedures. Melting points were determined on a Gallenkamp melting point apparatus and are uncorrected. IR spectra were registered on a Perkin Elmer FTIR-1600. ${ }^{1} \mathrm{H}$ NMR (300 MHz) and ${ }^{13} \mathrm{C}$ NMR (75.4 MHz) spectra were recorded on a Varian Unity

Plus Spectrometer. Double resonance, HMQC and HMBC experiments were carried out for complete assignment of proton and carbon signals in the NMR spectra, whenever possible. High resolution mass spectra were determined on a AutoSpec E spectrometer. Elemental analyses were obtained on a Leco CHNS-932 instrument. Compound 9 was prepared by a known method. ${ }^{[1]}$

\section{General procedure for preparation of $2 \mathrm{a}-\mathbf{d}$}

To a solution of 5-amino-4-cyano-1-substituted pyrazole 1a-d $(0.2 \mathrm{~mol})$, in ethanol (20 $\mathrm{mL})$ and triethylamine $(2 \mathrm{~mL})$ was heated under reflux for $7 \mathrm{~h}$ and then concentrated under reduced pressure. The solid product so formed was collected by filtration, washed with ethanol and crystallized from $\mathrm{EtOH}-\mathrm{H}_{2} \mathrm{O}$.

\section{Data}

6-(5-Amino-1-phenyl-1H-pyrazol-4-yl)-1-phenyl-1H-pyrazolo[3,4-d]pyrimidin-4-amine

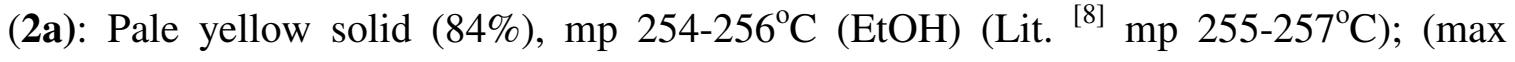
(Nujol mull): 3462 and 3302 (NH2) cm-1; $\delta \mathrm{H}$ (CDCl3): 5.57 (s, 2H, NH2-C-5”), 5.89 (s, 2H, NH $2-\mathrm{C}-4), 7.30-7.42$ (m, 2H, Ar-H), 7.52 (appt, 4H, 8.1 Hz, Ar-H), 7.60-7.67 (m, 2H, $\operatorname{Ar}-\mathrm{H}), 8.02$ (s, 1H, H-3“), $8.11(\mathrm{~d}, 2 \mathrm{H}, J=8.1 \mathrm{~Hz}, \mathrm{Ar}-\mathrm{H}), 8.23$ (s, 1H, H-3); $\delta_{\mathrm{C}}\left(\mathrm{CDCl}_{3}\right)$ : 99.04 (C-4“) 103.34 (C-3a), 121.72 (C-2`, C-6’or C-2”', C-6”'), 123.78 (C-2`, C-6’or C-2”"', C-6"'), 126.45 (C-4or C-4"'), 127.68 (C-4"'”or C-4'), 129.06 (C-3', C-5' or C-

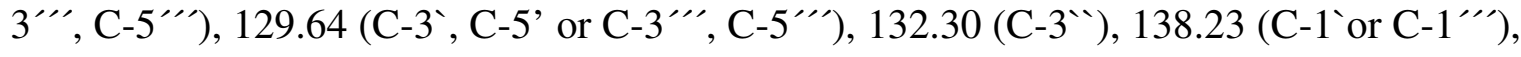
139.12 (C-1"' or C-1'), 141.03 (C-3), 146.89 (C-7a), 154.19 (C-5“), 157.14 (C-4), 160.42 (C-6). $m / z(\%) 368\left[\mathrm{M}^{+}\right]$(100). Anal. calcd. for $\mathrm{C}_{20} \mathrm{H}_{16} \mathrm{~N}_{8}$ (368.39): C, 65.21; $\mathrm{H}$, 4.38; N, 30.42. Found C, 65.10; H, 4.46; N, 29.99. 
6-(5-Amino-1-(4-chlorophenyl)-1H-pyrazol-4-yl)-1-(4-chlorophenyl)-1H-pyrazolo[3,4-d] pyrimidin-4-amine (2b): Pale yellow solid (72\%), mp 277-278 ${ }^{\circ} \mathrm{C}$ (EtOH-DMF); $v_{\max }$ (Nujol mull): 3463 and $3304\left(\mathrm{NH}_{2}\right) \mathrm{cm}^{-1} ; \delta_{\mathrm{H}}\left(\mathrm{DMSO}_{\mathrm{d}}\right): 6.89\left(\mathrm{~s}, 2 \mathrm{H}, \mathrm{NH}_{2}\right), 7.56-7.70$ (m, 6H, Ar-H), 7.92 (br s, 2H, NH 2 ), 8.08 (s, 1H, H-3 or H-3”), 8.28 (s, 1H, H-3 or H-3”), $8.30(\mathrm{~d}, 2 \mathrm{H}, J=9.0, \mathrm{Ar}-\mathrm{H})$. Anal. calcd. for $\mathrm{C}_{20} \mathrm{H}_{14} \mathrm{Cl}_{2} \mathrm{~N}_{8}$ (437.28): C, 54.93; H, 3.23; N, 25.62. Found C, 55.00; H, 3.23; N, 25.47.

6-(5-Amino-1-p-tolyl-1H-pyrazol-4-yl)-1-p-tolyl-1H-pyrazolo[3,4-d]pyrimidin-4-amine (2c): Pale yellow solid (78\%), mp 262-264 ${ }^{\circ} \mathrm{C}(\mathrm{EtOH}) ; v_{\max }(\mathrm{Nujol}$ mull): 3395, 3336, and $33187\left(\mathrm{NH}_{2}\right) \mathrm{cm}-1 ; \delta \mathrm{H}$ (DMSO-d6): $2.35\left(3 \mathrm{H}, \mathrm{s}, \mathrm{CH}_{3}\right), 2.36\left(3 \mathrm{H}, \mathrm{s}, \mathrm{CH}_{3}\right), 6.74(2 \mathrm{H}, \mathrm{s}$, $\mathrm{NH}_{2}$-C-4), 7.33 (2H, d, J=8.1 Hz, H-3', H5'or H-3“'”, H-5"'”), 7.34 (2H, d, J=8.1 Hz, H-

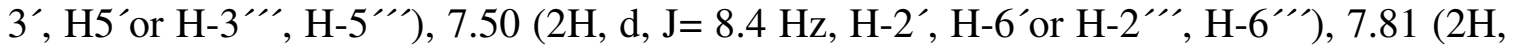
br s, $\left.\mathrm{NH}_{2}-\mathrm{C}-5 ”\right), 7.98$ (1H, s, H-3), 8.04 (2H, d, J= 8.4 Hz, H-2’, H-6’or H2"', H-6"'), $8.24\left(1 \mathrm{H}, \mathrm{s}, \mathrm{H}-3\right.$ ”); $\delta_{\mathrm{C}}\left(\mathrm{DMSO}_{6}\right): 20.66\left(\mathrm{CH}_{3}\right), 20.61\left(\mathrm{CH}_{3}\right), 98.58\left(\mathrm{C}-4{ }^{\prime \prime}\right), 102.40(\mathrm{C}-$ 3a), 120.58 (C-2', C-6'or C-2"'”, C-6"'), 123.24 (C-2`, C-6’or C-2”', C-6"') 129.65 (C-

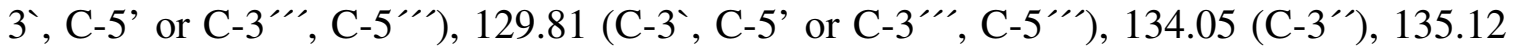

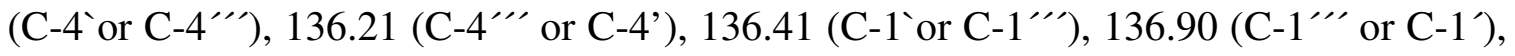
140.05 (C-3), 147.71 (C-7a), 153.82 (C-5“), 157.98 (C-4), 160.14 (C-6). Anal. calcd. for $\mathrm{C}_{22} \mathrm{H}_{20} \mathrm{~N}_{8} 21 \frac{1}{2} \mathrm{H}_{2} \mathrm{O}$ (441.45): C, 59.80; H, 4.53; N, 25.37. Found C, 59.82; H, 4.83; H, 25.06 .

6-(5-Amino-1-(4-bromophenyl)-1H-pyrazol-4-yl)-1-(4-bromophenyl)-1H-pyrazolo[3,4d] pyrimidin-4-amine (2d): Pale yellow solid (74\%), mp 284-285 ${ }^{\circ} \mathrm{C}$ (EtOH-DMF); $v_{\max }$ (Nujol mull): 3449, 3391 and $3301\left(\mathrm{NH}_{2}\right) \mathrm{cm}^{-1} ; \delta_{\mathrm{H}}\left(\mathrm{DMSO}-\mathrm{d}_{6}\right): 6.90\left(2 \mathrm{H}, \mathrm{s}, \mathrm{NH}_{2}\right), 7.33$ $(2 \mathrm{H}, \mathrm{d}, J=9.0 \mathrm{~Hz}, \mathrm{Ar}-\mathrm{H}), 7.69-7.78(4 \mathrm{H}, \mathrm{m}, \mathrm{Ar}-\mathrm{H}), 7.92\left(2 \mathrm{H}, \mathrm{br} \mathrm{s}, \mathrm{NH}_{2}\right), 8.09(1 \mathrm{H}, \mathrm{s}, \mathrm{H}-3$ or H-3” ), 8.26 (2H, d, J=9.0 Hz, Ar-H), 8.28 (1H, s, H-3” or H-3'). m/z(\%) (FAB+) 525 $\left[\mathrm{M}^{+}+1,{ }^{79} \mathrm{Br},{ }^{79} \mathrm{Br}\right](18), 527\left[\mathrm{M}^{+}+1,{ }^{79} \mathrm{Br},{ }^{81} \mathrm{Br}\right](32), 529\left[\mathrm{M}^{+}+1,{ }^{81} \mathrm{Br},{ }^{81} \mathrm{Br}\right]$ (19). Anal. calcd. for $\mathrm{C}_{20} \mathrm{H}_{14} \mathrm{Br}_{2} \mathrm{~N}_{8}$ (526.19): C, 45.65; H, 2.68; N, 21.30. Found C, 45.70; H, 2.75; N, 21.05 . 


\section{General procedure for preparation of $4 a, b$ and $6 a, b$}

A mixture of (1) (20 mmol) and malononitrile or diethylmalonate (20 mmol) was added to $20 \mathrm{~mL}$ freshly prepared sodium ethoxide solution [prepared by adding $1.0 \mathrm{~g}$ sodium metal into absolute ethanol $(20 \mathrm{~mL})]$ and the mixture was refluxed for $7 \mathrm{~h}$, and left to cool overnight. The solid product so formed was collected by filtration, washed with ethanol and crystallized from ethanol, unless otherwise stated.

\section{Data}

2-(4-Amino-1-phenyl-1H-pyrazolo[3,4-d]pyrimidin-6-yl)acetonitrile (4a): Pale yellow crystals $(77 \%) \mathrm{mp} 242-244^{\circ} \mathrm{C}(\mathrm{EtOH}) ; v_{\max }$ (Nujol mull): 3463 and $3296\left(\mathrm{NH}_{2}\right), 2213$ $(\mathrm{CN}) \mathrm{cm}^{-1} ; \delta_{\mathrm{H}}\left(\mathrm{DMSO}_{\mathrm{d}}\right): 4.17\left(\mathrm{~s}, 2 \mathrm{H}, \mathrm{CH}_{2}\right), 7.33\left(\mathrm{t}, 1 \mathrm{H}, J=7.5 \mathrm{~Hz}, \mathrm{H}-4^{\prime}\right), 7.54(\mathrm{t}, 2 \mathrm{H}$, $J=7.2 \mathrm{~Hz}, \mathrm{H}-3^{\prime}, \mathrm{H}^{\prime} 5^{\prime}$ ), 8.00 (br s, $1 \mathrm{H}, \mathrm{NH}_{2}$ ), 8.07 (br s, $2 \mathrm{H}, \mathrm{NH}_{2}$ ), 8.10-8.25 (br s, $1 \mathrm{H}$, $\mathrm{NH}_{2}$ ), $8.20\left(\mathrm{~d}, 2 \mathrm{H}, J=7.5 \mathrm{~Hz}, \mathrm{H}-2^{\prime}, \mathrm{H}-6^{\prime}\right), 8.34$ (s, 1H, H-3); $\delta_{\mathrm{C}}\left(\mathrm{DMSO}^{\prime} \mathrm{d}_{6}\right): 27.77$ $\left(\mathrm{CH}_{2}\right), 100.13$ (C-3a), $117.61(\mathrm{CN}), 120.45$ (C-2', C-6’), 124.12 (C-4'), 129.12 (C-3’', C5’), 134.13 (C-3), 138.86 (C-1`), 153.75 (C-7a), 158.34 (C-4), 159.67 (C-6). Anal. calcd. for $\mathrm{C}_{13} \mathrm{H}_{10} \mathrm{~N}_{6}$ (250.26): C, 62.39; H, 4.03; N, 33.58. Found C, 62.27; H, 4.22; N, 33.63.

2-[4-Amino-1-(4-chlorophenyl)-1H-pyrazolo[3,4-d]pyrimidin-6-yl]acetonitrile (4b): Yellow powder (81\%) mp 296-298 ${ }^{\circ} \mathrm{C}$ (EtOH-DMF); v $v_{\max }$ (Nujol mull): 3469 and 3306, $\left(\mathrm{NH}_{2}\right), 2218(\mathrm{CN}) \mathrm{cm}^{-1} ; \delta_{\mathrm{H}}\left(\mathrm{DMSO}_{-} \mathrm{d}_{6}\right): 4.17$ (s, 2H, $\left.\mathrm{CH}_{2}\right), 7.61$ (d, 2H, J = 9.3 Hz, H-3', H-5'), 8.04 (br s, 2H, NH ${ }_{2}$ ), 8.25 (d, 2H, $J=9.0$ Hz, H-2’' H-6’), 8.37 (s, 1H, H-3); $\delta_{\mathrm{C}}$ (DMSO-d $\left.{ }_{6}\right): 27.81\left(\mathrm{CH}_{2}\right), 100.23$ (C-3a), 117.63 (CN), 121.82 (C-2`', C-6'), 129.18 (C-3', C-5'), 130.22 (C-4’), 134.63 (C-3), 137.75 (C-1`), 153.89 (C-7a), 158.37 (C-4), 159.90 (C-6). $m / z(\%)$ (TOF) $284\left[\mathrm{M}^{+},{ }^{35} \mathrm{Cl}\right]$ (100), $286\left[\mathrm{M}^{+},{ }^{37} \mathrm{Cl}\right]$ (18). $\mathrm{C}_{13} \mathrm{H}_{9} \mathrm{ClN}_{6}$ (284.70): C, 54.84; H, 3.19; N, 29.52. Found C, 54.78; H, 3.08; N, 29.42.

Ethyl 4-amino-1-(phenyl)-6-hydroxy-1H-pyrazolo[3,4-b]pyridine-5-carboxylate (6a): White powder $(89 \%) \mathrm{mp} 197-199^{\circ} \mathrm{C}(\mathrm{EtOH})$; $v_{\max }$ (Nujol mull): 3505 and $3395\left(\mathrm{NH}_{2}\right)$, 3245 (br, OH), $1660(\mathrm{C}=\mathrm{O}) \mathrm{cm}^{-1}$; $\delta_{\mathrm{H}}\left(\mathrm{DMSO}_{\mathrm{d}}\right): 1.34$ (t, $\left.3 \mathrm{H}, J=7.5 \mathrm{~Hz}, \mathrm{CH}_{3}\right), 4.38(\mathrm{q}$, $\left.2 \mathrm{H}, J=7.5 \mathrm{~Hz}, \mathrm{CH}_{2}\right), 7.31\left(\mathrm{t}, 1 \mathrm{H}, J=7.5 \mathrm{~Hz}, \mathrm{H}^{\prime} 4^{\prime}\right), 7.51$ (t, 2H, $\left.J=7.5 \mathrm{~Hz}, \mathrm{H}-3^{\prime}\right), 8.06$ (d, 4H, $J=7.8 \mathrm{~Hz}, \mathrm{H}-2^{\prime}, \mathrm{H}-6^{\prime}, \mathrm{NH}_{2}$ ), 8.44 (s, 1H, H-3), 12.32 (br s, $1 \mathrm{H}, \mathrm{OH}$ ); $\delta_{\mathrm{C}}$ 
(DMSO-d $\left.{ }_{6}\right): 14.30\left(\mathrm{CH}_{3}\right), 61.24\left(\mathrm{CH}_{2}\right), 86.57$ (C-5), 102.09 (C-3a), 121.01 (C-2`, C-6'), 126.05 (C-4'), 129.05 (C-3`, C-5’), 135.12 (C-3), 138.87 (C-1`), 148.95 (C-7a), 152.76 (C-4), 165.99 (C-6), $170.33(\mathrm{C}=\mathrm{O})$. Anal. calcd. for $\mathrm{C}_{15} \mathrm{H}_{14} \mathrm{~N}_{4} \mathrm{O}_{3}$ (298.30): C, 60.40; $\mathrm{H}$, 4.73; N, 18.78. Found C, 59.99; H, 4.93; N, 18.58.

Ethyl 4-amino-1-(4-chlorophenyl)-6-hydroxy-1H-pyrazolo[3,4-b]pyridine-5-carboxylate (6b): White powder (82\%) mp 314-315 ${ }^{\circ} \mathrm{C}$ (EtOH-DMF); $v_{\max }$ (Nujol mull): 3486 and $3363\left(\mathrm{NH}_{2}\right), 3179(\mathrm{br}, \mathrm{OH}), 1687(\mathrm{C}=\mathrm{O}) \mathrm{cm}^{-1} ; \delta_{\mathrm{H}}\left(\mathrm{DMSO}_{6} \mathrm{~d}_{6}\right): 1.32(\mathrm{t}, 3 \mathrm{H}, J=7.5 \mathrm{~Hz}$, $\left.\mathrm{CH}_{3}\right), 4.36\left(\mathrm{q}, 2 \mathrm{H}, J=7.5 \mathrm{~Hz}, \mathrm{CH}_{2}\right), 7.55(\mathrm{~d}, 2 \mathrm{H}, J=7.0 \mathrm{~Hz}, \mathrm{Ar}-\mathrm{H}), 7.95\left(\mathrm{~s}, 2 \mathrm{H}, \mathrm{NH}_{2}\right)$, 8.20 (d, 2H, $J=7.0 \mathrm{~Hz}$, Ar-H), 8.40 (s, 1H, H-3), 11.80-12.90 (br s, 1H, OH). m/z(\%) (TOF) $332\left[\mathrm{M}^{+},{ }^{35} \mathrm{Cl}\right](16), 334\left[\mathrm{M}^{+},{ }^{37} \mathrm{Cl}\right]$ (5). Anal. calcd. for $\mathrm{C}_{15} \mathrm{H}_{13} \mathrm{ClN}_{4} \mathrm{O}_{3}$ (332.74): $\mathrm{C}$, 54.14; H, 3.94; N, 16.84. Found C, 54.25; H, 4.09; N, 16.66.

\section{General procedure for preparation of 8a, b}

A mixture of (1) (20 mmol) and phosphoryl chloride $(3.83 \mathrm{~g}, 25 \mathrm{mmol})$ in anhydrous DMF ( $5 \mathrm{~mL}$ ) was heated under stirring at $70^{\circ} \mathrm{C}$ for $3 \mathrm{~h}$. Then, the reaction mixture was poured onto ice and treated with aqueous ammonia ( $\mathrm{pH} \mathrm{8).} \mathrm{A} \mathrm{white} \mathrm{solid} \mathrm{separated} \mathrm{and} \mathrm{it}$ was filtered off, washed with water, dried and recrystallized from an appropriate solvent to afford the products in $60-82 \%$ yields.

1-Phenyl-1H-pyrazolo[3,4-d]pyrimidin-4-ol (8a): White powder (70\%) mp 294-296 ${ }^{\circ} \mathrm{C}$ $\left(\right.$ EtOH-DMF), (Lit. mp 299 $\left.{ }^{\circ} \mathrm{C}[11]\right) \delta_{\mathrm{H}}\left(\mathrm{DMSO}_{-} \mathrm{d}_{6}\right): 7.38$ (t, 1H, J= $\left.7.8 \mathrm{~Hz}, \mathrm{H}-4^{\prime}\right), 7.54$ (t, 2H, $J=7.8 \mathrm{~Hz}, \mathrm{H}-3^{\prime}$ and H-5'), 8.03 (d, 2H, $J=7.5 \mathrm{~Hz}, \mathrm{H}-2^{\prime}, \mathrm{H}-6^{\prime}$ ), 8.19 (s, 1H, H-6), 8.32 (s, 1H, H-3), 12.46 (br s, 1H, OH); $\delta_{\mathrm{C}}\left(\mathrm{DMSO}_{6}\right)$ ) 107.62 (C-3a), 121.75 (C-2), C6’), 127.13 (C-4'), 129.22 (C-3’, C-5’), 136.00 (C-3), 132.22 (C-1'), 148.81 (C-6), 151.85 (C-7a), 157.23 (C-4). Anal. calcd. for $\mathrm{C}_{11} \mathrm{H}_{8} \mathrm{~N}_{4} \mathrm{O}$ (212.21): C, 62.26; H, 3.80; N, 26.40. Found C, 61.89; H, 3.87; N, 26.75.

1-(4-Chlorophenyl)-1H-pyrazolo[3,4-d]pyrimidin-4-ol (8b): White powder (74\%) mp 314-316 ${ }^{\circ} \mathrm{C}\left(\right.$ EtOH-DMF), (Lit. mp $\left.>300^{\circ} \mathrm{C}[11]\right) \delta_{\mathrm{H}}\left(\right.$ DMSO-d $\left._{6}\right): 7.63$ (d, 2H, $J=9.3 \mathrm{~Hz}$, Ar-H), 8.10 (d, 2H, J = 9.0 Hz, Ar-H), 8.22 (s, 1H, H-6), 8.35 (s, 1H, H-3), 12.30-12.70 
(br s, 1H, OH). Anal. calcd. for $\mathrm{C}_{11} \mathrm{H}_{7} \mathrm{ClN}_{4} \mathrm{O}$ (246.65): C, 53.56; H, 2.86; N, 22.71 . Found C, 53.16; H, 3.04; N, 22.55.

5-Amino-1-(4-chlorophenyl)-1H-pyrazole-4-carboxamide (9): White powder (81\%), mp 202-204 ${ }^{\circ} \mathrm{C}(\mathrm{EtOH}),\left(\mathrm{Lit} \mathrm{mp} 204-205^{\circ} \mathrm{C},[11]\right), v_{\max }$ (Nujol mull): 3471, 3337, $\left(\mathrm{NH}_{2}\right)$, $1662(\mathrm{C}=\mathrm{O}) \mathrm{cm}-1 ; \delta_{\mathrm{H}}\left(\mathrm{CDCl}_{3}\right): 6.42\left(\mathrm{~s}, 2 \mathrm{H}, \mathrm{NH}_{2}\right), 6.857$ (br s, 1H, NH), 7.41 (br s, 1H, $\mathrm{NH})$, 7.54-7.57 (m, 4H, Ar-H), 7.90 (s, 1H, H-3); $\delta_{\mathrm{C}}\left(\mathrm{CDCl}_{3}\right)$ : 97.65 (C-4), 124.75 (C-2), C-6’), 129.30 (C-3`, C-5’), 131.21 (C-4`), 137.13 (C-1`), 139.30 (C-3), 149.48 (C-5), $166.03(\mathrm{C}=\mathrm{O}) \cdot \mathrm{m} / \mathrm{z}(\%)$ (TOF) $236\left[\mathrm{M}^{+},{ }^{35} \mathrm{Cl}\right](30), 238\left[\mathrm{M}^{+},{ }^{37} \mathrm{Cl}\right]$ (6). Anal. calcd. for $\mathrm{C}_{10} \mathrm{H}_{9} \mathrm{ClN}_{4} \mathrm{O}$ (236.66): C, 50.75; H, 3.83; N, 23.67. Found C, 51.21; H, 3.91; N, 23.71.

\section{ACKNOWLEDGEMENTS}

We thank Fundação para a Ciência e Tecnologia and FEDER (POCTI-SFA-3-686) and post-doctoral grant for A. Salaheldin (SFRH/BPD/31490/2006) and Miss Elisa Pinto for obtaining the NMR and elemental analyses data.

\section{REFERENCES}

1. Hamilton, H. W.; Ortwine, D. F.; Worth, D. F.; Bristol, J. A. Synthesis and structureactivity relationships of pyrazolo[4,3- $d]$ pyrimidin-7-ones as adenosine receptor antagonists. J. Med. Chem. 1987, 30, 91-96.

2. Poulsen, S. A.; Quinn, R. J. Synthesis and Structure-Activity Relationship of Pyrazolo[3,4- $d$ ]pyrimidines: Potent and Selective Adenosine $\mathrm{A}_{1}$ Receptor Antagonists. J. Med. Chem. 1996, 39, 4156-4161.

3. Harden, F. A.; Quinn, R. J.; Scammells, P. J. Synthesis and adenosine receptor affinity of a series of pyrazolo[3,4- $d]$ pyrimidine analogs of 1-methylisoguanosine. J. Med. Chem. 1991, 34, 2892-2898.

4. Lynck, B. M.; Khan, M. A.; Teo, H. C.; Pedrotti, F. Pyrazolo[3,4-b]pyridines: Syntheses, reactions, and nuclear magnetic resonance spectra. Can. J. Chem. 1988, $66,420-428$. 
5. Salaheldin, A. M.; Campos, A. M.; Rodrigues, L. M. N-Bromosuccinimide assisted oxidation of 5-aminopyrazoles: formation of bis diazenylderivatives. Tetrahedron Lett. 2007, 48, 8819-8822.

6. Campos, A. M.; Salaheldin, A. M.; Rodrigues, L. M. Synthesis of some novel pyrazolo[3,4-d]pyrimidine derivatives. Arkivoc, 2007, xvi, 92-100.

7. Gupta S.; Rodrigues, L. M.; Esteves, A. P.; Oliveira-Campos, A. M. F.; Nascimento, M. S. J.; Nazareth, N.; Cidade, H.; Neves, M. P.; Pinto, E. F. M.; Cerqueira, N. M. F. and Brás N. Synthesis of $\mathrm{N}$-aryl-5-amino-4-cyanopyrazole derivatives as potent xanthine oxidase inhibitors. Eur. J. Med. Chem. (Article in Press) doi:10.1016/j.ejmech.2007.06.002.

8. Taylor, E. D.; Borror, A. L. The reaction of Nitriles with o-Aminonitriles: Aconvenient synthesis of fused 4-Aminopyrimidine. J. Org. Chem. 1961, 26, 4967-4974.

9. Smyrl, N. R.; Smithwick, R. W. Hydroxide-catalyzed synthesis of heterocyclic aromatic amine derivative from nitriles. J. Heterocyclic Chem. 1982, 19, 493-496.

10. Veronese, A. C.; Callegari, R.; Salah, S. A. Tin (VI) chloride-promoted reaction of $\beta$ dicarbonyl compounds with nitrile. Synthesis of aminopyridines and aminoquinolines. Tetrahedron. Lett. 1990, 31, 3485-3488.

11. Cheng, C. C.; Robins, R. K. Potential purine antagonists. VI. Synthesis of 1-Alkyland 1-Aryl-4-aubatituted pyrazolo[3,4-d]pyrimidines. J. Org. Chem. 1956; 21; 12401256. 